\title{
Digging into the Details of Cells and Tissues Using 3D ToF-SIMS Imaging
}

\section{Daniel Graham and Lara Gamble}

University of Washington, Seattle, Washington, United States

Time-of-flight secondary ion mass spectrometry (ToF-SIMS) generates spectra rich in chemical information from the near surface region. Modern ToF-SIMS instruments can generate 2D images where every pixel in the spectrum contains a full mass spectrum with image resolutions down to $\sim 100 \mathrm{~nm}(16 \%$ $84 \%$ edge resolution). ToF-SIMS instruments are capable of generating images that can isolate single cell layers and clearly show structural and chemical information from single cells. With the addition of new gas cluster ion sputter sources, ToF-SIMS can also generate detailed chemical data in 3D with depth resolutions down to a few nanometers. Using these strengths, we have employed 3D ToF-SIMS imaging for the characterization of cell and tissue samples.

A combination of ToF-SIMS and AFM have been used to demonstrate that ToF-SIMS 3D depth profiles accurately represent the shape and topography of single cells in 3D[1]. To accurately reconstruct ToFSIMS data in 3D, the data was reconstructed using the ZCorrectroGUI from our NBToolbox (NBToolbox, https://www.nb.uw.edu/mvsa/nbtoolbox). Additionally, utilizing accurately reconstructed 3D chemical images of cells, we showed localized polymer nanoparticles within cells could be imaged. This presented a particular challenge as polymers typically consist of the same elements and chemical groups that exist within cells. The result of this is that signals typically thought to be characteristic of a given polymer often overlap with signals from the cells. To circumvent this issue, we developed a background subtraction method wherein we subtracted the background signal from cells that did not contain nanoparticles from cells that did contain nanoparticles. Using this method we show that we were able to localize polymer nanoparticles that were designed to be trapped within endosomes[2]. Localization of the nanoparticles in endosomes was validated using confocal microscopy of cells treated with the same nanoparticles.

The ability to generate accurate 3D chemical images can enable detailed analysis of biological samples. However, working with biological samples can be difficult and we will highlight some of these challenges with examples from 3D ToF-SIMS imaging. This methodology has the potential to provide important information about not only the localization and distribution of drug molecules, nanoparticles and metabolites, but also their effect on the chemical environment with cells and tissues. This can provide complementary information to data generated from other imaging modalities and help provide a more complete chemical picture of biomaterials and biological samples.

\section{References}

1. Robinson, M. A., Graham, D. J., and Castner D. G., "ToF-SIMS Depth Profiling of Cells: z-Correction, 3D Imaging, and Sputter Rate of Individual NIH/3T3 Fibroblasts", Analytical Chemistry, 2012, 84, 48804885.

2. Daniel J. Graham, John T. Wilson, James J. Lai, Patrick S. Stayton, and David G. Castner, Threedimensional localization of polymer nanoparticles in cells using ToF-SIMS. Biointerphases, 2016, 11, $02 \mathrm{~A} 304$. 